Punjab University Journal of Mathematics (2021),53(10),691-710

https://doi.org/10.52280/pujm.2021.531001

\title{
Multipolar Interval-Valued Fuzzy Set with Application of Similarity Measures and multi-person TOPSIS technique
}

\author{
Muhammad Saeed, Asad Mehmood and Muhammad Arslan \\ Department of Mathematics, University of Management and Technology, Lahore, Pakistan \\ Email: muhammad.saeed@umt.edu.pk*, a.asadkhan.khi@gmail.com, \\ arslanrao233@gmail.com
}

Received: 12 April,2021 / Accepted: 18 September, 2021 / Published online: 25 October , 2021

\begin{abstract}
A Similarity measure in the fuzzy structure plays a very considerable role in manipulating hurdles that apprehend vague data, but unable to deal with the ambiguous and variability of the problems having multipolar interval-valued data. In this research article, a certain distance between two multipolar interval-valued fuzzy sets ( $m$ IVF sets) has been defined. A new similarity measure (Sim.M) for $\mathrm{mIVF}$ based on distances has been introduced, also some of the basic operations on the structure has been defined such as union, intersection, and complement. MCDM is performed for $m \mathrm{IVF}$ information that measure the similarity measure based on distance measure for the best alternative. An application is given that the proposed Sim.M for $m$ IVF set is capable of recognition the nature and structure of different entities which belongs to the same family. Furthermore, a multiperson TOPSIS technique is developed for the structure of $m$ IVF with an algorithm for the selection of the best alternative.
\end{abstract}

Key Words: Multipolar Interval-valued Fuzzy Set ( $m$ IVF set), Operators and Properties, Distance measure and similarity measure (Sim.M), Pattern Recognition, multi-person TOPSIS technique.

\section{INTRODUCTION}

The scientific models of crisp set logic and theory are not sufficient to carry out the problems that contains hesitancy. Zadeh [41] defined fuzzy set logic as the generalisation crisp set theory, emphasizing the complexities of these structure with the membership approach. Atanassov [10] defined an intuitionistic fuzzy set by separately assigning membership and non-membership function. Zadeh [42], Grattan [17], and Jahn [20] individualistically established the interval-valued fuzzy set (IVF)in the same year. IVF is defined by an association function interval-valued about membership. An Interval-valued fuzzy set is an unusual case of L-fuzzy sets in the Goguen sense [18], so as a mathematical object, it is not of exact interest. 
Zhang [26] introduced the novel idea to deal the vagueness data in two different opinion as bipolar fuzzy sets. Chen [12] presented the concept of multipolar fuzzy sets as a simplified type of fuzzy bipolar set. Akram ([2]-[8],[1]) introduced mpolar fuzzy graph, characterization of mpolar fuzzy graph and metrics in mpolar fuzzy graph. Riaz([28]-[29]) proposed the idea of bipolar fuzzy soft set topology and fuzzy parameterized fuzzy soft set metric spaces in decision making for the world problem. Rashid ([27]-[11]) inherited the experimental verification using fuzzy logic modeling on used foundry sand, also measure the similarity between intuitionistic fuzzy through inclusion.

Chen ([15]-[13]) gave the idea of Similarity Measure of unknown sets, but some problems had been unable to be contained by it. To overcome with this problem, Kim and Hong [19] proposed several new updated steps. The Interval-valued fuzzy set was firstly proposed by Gorzalczany [16] in 1987, also defines their basic operations including union, intersection, and complement. Kharal [21] presented some similarity measure based on set-theoretical operations. Akram [9] implemented a multipolar fuzzy set and multipolar fuzzy soft software similarity measures by application of pattern recognition and medical diagnosis. riaz et al. [44] studied the TOPSIS approach in exploring most effectual method for curing from COVID-19 in Pythagorean m-polar fuzzy topological structure. Young [39] proposed a new method for determining the best location for a plantation in terms of the linguistic structure, using graded mean reparation and fuzzy logic. Olson's work [24] discusses the multiple criteria for identification of solution in TOPSIS and take its comparison with SMART and centroid weighting schemes. Chen [14] describes the TOPSIS to the fuzzy environment in the triangular sense in 2000. Hsu [38] explained the strategy of order choice by similarities using MADM in a TOPSIS-based group decision setting. Furthermore, Similarity measure and a TOPSIS technique is used by Saeed ([30]-[33]). Application of fuzzy numbers in mobile selection in metros like Lahore is proposed by Saqlain [37]. Application of similarity measure on multipolar structure is discussed by Saeed et al. [34] in medical diagnosis and decision-making. Mehmood et al. ([35],[36]) applied the distance based similarity measure in the spread recognition of COVID-19 in Pakistan with the correspondence to top 16 affected countries and extented the fuzzyy TOPSS to intuinistic fuzzy environment under linguistic variable and triangular numbers. Khalifa et al. [22] discussed new decision making technique with the application in mass media. Petrovic and Kankaras [25] developed a hybridized interval type-2 fuzzy sets DEMATEL,AHP and TOPSIS multicriteria decision making approach for the selection and evaluation of criteria for determination of air traffic control radar position. Yorulmaz et al. [40] discussed the robust mahalanobis distance based TOPSIS to evaluate the economic development of 81 provinces of Turkey. Zolfani et al. [43] studied VIKOR and TOPSIS focused reanalysis of the MADM methods based on logarithmic normalization.

1.1. Motivation. This article extends the idea of $m \mathrm{~F}$ set and interval-valued fuzzy set into $m I V F$ set, the reason behind this extension is the existence of the multipolar interval-valued fuzzy data because many real world problems are in the form of $\mathrm{m}$-attributes expressed in interval valued rather than single-valued form. Existing theories like fuzzy set discussed by Zadeh in [41], bipolar fuzzy set discussed by Wen-Ran in [26], multipolar fuzzy set discussed by Akram et al. in [9] and Interval-valued discussed by Jahn in [20] are inadequate for this purpose as they don't utilize data comprehensively in the form multi-interval-valued 
fuzzy. The persistence of this article is to the association of the interval-valued fuzzy set (IVF set) and $m \mathrm{~F}$ set to acquire a new fuzzy set model: multipolar Interval-valued fuzzy set. The goal of this research is to develop a suitable framework $m$ IVF and investigates the distance and similarity measures on $m$ IVF. Furthermore, for this new developed structure $m I V F$, a multi-person TOPSIS technique has been presented to get a better and accurate results with the evaluation of multi experts in the related field of any decision analysis.

1.2. Structure of Paper. To smooth our discussion, at first in Section 2, we extant some fundamentals related to this article. In Section 3, the notion of multipolar interval-valued fuzzy ( $m$ IVF) set and its basic operations are defined. In Section 4, some properties based on operators are studied. Section 5 discusses the distance measure formulas for the $m \mathrm{IVF}$ set. In Section 6, Similarity Measure based on distance has been introduced. Section 7 has application of similarity measure on the $m \mathrm{IVF}$ set in pattern recognition of a watch brand with the algorithm. In section 8 , a TOPSIS technique is developed for the multiperson decision analysis on the structure of $m \mathrm{IVF}$ set. A hypothetical data numerical example is considered for the evaluation of proposed multiperson TOPSIS technique. After that, the article concludes with an analysis and and future work.

\section{Preliminaries}

This section discusses some basic definitions related to the $m$ IVF Set.

Definition 2.1 (Fuzzy Set). [1] A Fuzzy Set $Q$ over universal set $Y$ is defined as

where the mapping $\mu_{Q}: Y \rightarrow[0,1]$,

$$
Q=\left\{\left(y, \mu_{Q}(y)\right): y \in Y\right\}
$$

Definition 2.2 ( $m \mathrm{~F}$ Set). [3] An $m F$ set over universal set $Y$ is a mapping $T=\left(z_{1} \circ T(y)\right.$, $\left.z_{2} \circ T(y), \cdots, z_{m} \circ T(y)\right): Y \rightarrow[0,1]^{m}$ where the $i$-th projection mapping is defined $z_{i} \circ T:[0,1]^{m} \rightarrow[0,1]$.

Definition 2.3 (Interval Valued Fuzzy Set). [15] An Interval-valued Fuzzy set $Q$ over universal set $Y$ is defined as

$$
Q=\left\{\left(y, I_{Q}(y)\right): y \in Y\right\}
$$

where,

$$
I_{Q}(y) \subseteq[0,1]
$$

\section{Multipolar Interval-Valued Fuzzy Set and Some Operations defined} ON $m$ IVF SETS

Definition 3.1 (Multipolar Interval-Valued Fuzzy Set). An mIVF set $Q$ over nonempty universal set $Y$, is a function

$$
Q=\left\{y,\left(p_{1} \circ I_{Q}(y), p_{2} \circ I_{Q}(y), \cdots, p_{m} \circ I_{Q}(y)\right)\right\},
$$

where $i$-th projection mapping is

$$
\begin{aligned}
& p_{i} \circ I_{Q}(y) \subseteq[0,1] \\
& \text { for all } i=1,2, \cdots, m
\end{aligned}
$$

Example 3.1 2-polar IVF set over the universal set

$Y=\left\{y_{1}, y_{2}\right\}$ is expresses as 


$$
R=\left\{\left(y_{1},[0.2,0.3],[0,2,0.4]\right),\left(y_{2},[0.4,0.45],[0.5,0.6]\right)\right\}
$$

Definition 3.2 ( $m$ IVF Subset). Let $Q$ and $R$ be two mIVF set over universal set $Y$ and defined as

$$
\begin{gathered}
Q=\left\{y, p_{i} \circ I_{Q}(y): y \in Y\right\} \text { and } R=\left\{y, p_{i} \circ I_{R}(y): y \in Y\right\} \\
\text { for all } i=1,2, \cdots, m
\end{gathered}
$$

where,

$$
\begin{gathered}
p_{i} \circ I_{Q}(y)=\left[p_{i} \circ I_{Q}^{L}(y), p_{i} \circ I_{Q}^{U}(y)\right], \\
p_{i} \circ I_{R}(y)=\left[p_{i} \circ I_{R}^{L}(y), p_{i} \circ I_{R}^{U}(y)\right] \\
\text { for all } i=1,2, \cdots, m
\end{gathered}
$$

$Q$ is subset of $R$ if $p_{i} \circ I_{Q}(y)$ is contained in $p_{i} \circ I_{R}(y)$. i.e.

$$
\begin{gathered}
p_{i} \circ I_{Q}^{L}(y) \geq p_{i} \circ I_{R}^{L}(y) \text { and } p_{i} \circ I_{Q}^{U}(y) \leq p_{i} \circ I_{R}^{U}(y) \\
\qquad \text { for all } i=1,2, \cdots, m
\end{gathered}
$$

Example 3.2 $Q$ and $R$ are 2-polar IVF sets over universal set $Y$.

$$
\begin{aligned}
& Q=\left\{\left(y_{1},[0.2,0.3],[0,4,0.5]\right),\left(y_{2},[0.3,0.6],[0.4,0.5]\right)\right\} \\
& R=\left\{\left(y_{1},[0.1,0.4],[0,4,0.6]\right),\left(y_{2},[0.2,0.6],[0.4,0.5]\right)\right\}
\end{aligned}
$$

here $Q$ is subset of $R$.

Definition 3.3 ( $m$ IVF Equal set). Let $Q=\left\{y, p_{i} \circ I_{Q}(y): y \in Y\right\}$ and $R=\left\{y, p_{i} \circ I_{R}(y): y \in Y\right\}$ be the two mIVF sets over the universal set $Y$ are equal if $Q \hat{\subseteq} R$ such that $p_{i} \circ I_{Q}(y)$ is contained in $p_{i} \circ I_{R}(y)$

and

$$
R \hat{\subseteq} Q \text { such that } p_{i} \circ I_{R}(y) \text { is contained in } p_{i} \circ I_{Q}(y)
$$

then

$$
Q=R
$$

Definition 3.4 ( $m$ IVF Null Set). A set $Q$ is said to be $m I V F$ null set, denoted by $\phi$ if

$$
\begin{gathered}
Q=\left\{\left(y, p_{i} \circ I_{Q}(y)=[1,0]\right): y \in Y\right\}=\phi \\
\text { for all } i=1,2, \cdots, m
\end{gathered}
$$

Definition 3.5 ( $m$ IVF Absolute Set). A set $R$ is said to be $m I V F$ absolute set, denoted by $Y$ if

$$
\begin{gathered}
R=\left\{\left(y, p_{i} \circ I_{R}(y)=[0,1]\right): y \in Y\right\}=Y \\
\quad \text { for all } i=1,2, \cdots, m
\end{gathered}
$$

Definition 3.6 (Union of $m I \mathrm{VF}$ Sets). Let $Q=\left\{y, p_{i} \circ I_{Q}(y): y \in Y\right\}$ and $R=\left\{y, p_{i} \circ I_{R}(y): y \in Y\right\}$ be two mIVF sets over the universal set $Y$ where,

$$
\begin{gathered}
p_{i} \circ I_{Q}(y)=\left[p_{i} \circ I_{Q}^{L}(y), p_{i} \circ I_{Q}^{U}(y)\right], \\
p_{i} \circ I_{R}(y)=\left[p_{i} \circ I_{R}^{L}(y), p_{i} \circ I_{R}^{U}(y)\right] \\
\text { for all } i=1,2, \cdots, m
\end{gathered}
$$

then their union is defined as, 


$$
\begin{gathered}
Q \hat{\cup} R=\left\{y,\left[\min \left(p_{i} \circ I_{Q}^{L}(y), p_{i} \circ I_{R}^{L}(y)\right), \max \left(p_{i} \circ I_{Q}^{U}(y), p_{i} \circ I_{R}^{U}(y)\right)\right]\right\} \\
\text { for all } i=1,2, \cdots, m
\end{gathered}
$$

Definition 3.7 (Intersection of $m$ IVF Sets). Let $Q$ and $R$ be are two mIVF set over $Y$ defined as

$$
\begin{gathered}
Q=\left\{y, p_{i} \circ I_{Q}(y): y \in Y\right\} \text { and } R=\left\{y, p_{i} \circ I_{R}(y): y \in Y\right\} \\
\text { for all } i=1,2, \cdots, m
\end{gathered}
$$

where,

$$
\begin{gathered}
p_{i} \circ I_{Q}(y)=\left[p_{i} \circ I_{Q}^{L}(y), p_{i} \circ I_{Q}^{U}(y)\right] \\
p_{i} \circ I_{R}(y)=\left[p_{i} \circ I_{R}^{L}(y), p_{i} \circ I_{R}^{U}(y)\right] \\
\text { for all } i=1,2, \cdots, m
\end{gathered}
$$

then, their intersection is given by

$$
\begin{gathered}
Q \hat{\cap} R=\left\{y,\left[\max \left(p_{i} \circ I_{Q}^{L}(y), p_{i} \circ I_{R}^{L}(y)\right), \min \left(p_{i} \circ I_{Q}^{U}(y), p_{i} \circ I_{R}^{U}(y)\right)\right]\right\} \\
\text { for all } i=1,2, \cdots, m
\end{gathered}
$$

Definition 3.8 (Complement). Complement of a mIVF set $Q=\left\{\left(y, p_{i} \circ I_{Q}(y)\right): y \in Y\right\}$ can be defined as

$$
\begin{gathered}
Q^{c}=\left\{y,\left([0,1]-p_{i} \circ I_{Q}(y)\right): y \in Y\right\} \\
\text { for all } i=1,2, \cdots, m
\end{gathered}
$$

\section{Example 3.3 If}

$$
\begin{gathered}
Q=\left\{\left(y_{1},[0.2,0.3],[0,4,0.5]\right),\left(y_{2},[0.3,0.6],[0.4,0.5]\right)\right\} \text { and } \\
R=\left\{\left(y_{1},[0.3,0.5],[0,2,0.4]\right),\left(y_{2},[0.3,0.5],[0.2,0.5]\right)\right\}
\end{gathered}
$$

be the 2 - polar interval valued fuzzy set over universal set $Y=\left\{y_{1}, y_{2}\right\}$, then their union, intersection, and complement will be respectively,

$$
\begin{aligned}
& Q \hat{\cup} R=\left\{\left(y_{1},[0.2,0.5],[0,2,0.5]\right),\left(y_{2},[0.3,0.6],[0.2,0.5]\right)\right\}, \\
& Q \hat{\cap} R=\left\{\left(y_{1},[0.3,0.3],[0.4,0.4]\right),\left(y_{2},[0.3,0.5],[0.4,0.5]\right)\right\},
\end{aligned}
$$

$Q^{c}=\left\{\left(y_{1},[0,0.2) \cup(0.3,1],[0,0.4) \cup(0.5,1]\right),\left(y_{2},[0,0.3) \cup(0.6,1],[0,0.4) \cup(0.5,1]\right)\right\}$.

\section{PRoperties of $m$ IVF SETS OPERATIONS}

Some of the major properties of set-theoretic operators defined on $m$ IVF sets are discussed below.

Idempotent Properties 4.1 Idempotent properties hold true for an $m$ IVF set $Q$ over universal set $Y$, can be defined as

and

$$
Q \hat{\cup} Q=\left\{y,\left[\min \left(p_{i} \circ I_{Q}^{L}(y), p_{i} \circ I_{Q}^{L}(y)\right), \max \left(p_{i} \circ I_{Q}^{U}(y), p_{i} \circ I_{Q}^{U}(y)\right)\right]\right\}=Q
$$

$$
Q \hat{\cap} Q=\left\{y,\left[\max \left(p_{i} \circ I_{Q}^{L}(y), p_{i} \circ I_{Q}^{L}(y)\right), \min \left(p_{i} \circ I_{Q}^{U}(y), p_{i} \circ I_{Q}^{U}(y)\right)\right]\right\}=Q
$$


Identity Properties 4.2 Identity properties also hold true for an $m$ IVF set $Q$ over universal set $Y$ as

and

$$
Q \hat{\cup} \phi=\left\{y,\left[\min \left(p_{i} \circ I_{Q}^{L}(y), 1\right), \max \left(p_{i} \circ I_{Q}^{U}(y), 0\right)\right]\right\}=Q
$$

$$
\begin{gathered}
Q \hat{\cap} Y=\left\{y,\left[\max \left(p_{i} \circ I_{Q}^{L}(y), 0\right), \min \left(p_{i} \circ I_{Q}^{U}(y), 1\right)\right]\right\}=Q \\
\text { for all } i=1,2, \cdots, m
\end{gathered}
$$

Domination Properties 4.3 Domination properties for a $m$ IVF set $Q$ over universal set $Y$ is given as

and

$$
Q \hat{\cup} Y=\left\{y,\left[\min \left(p_{i} \circ I_{Q}^{L}(y), 0\right), \max \left(p_{i} \circ I_{Q}^{U}(y), 1\right)\right]\right\}=Y
$$

$$
Q \hat{\cap} \phi=\left\{y,\left[\max \left(p_{i} \circ I_{Q}^{L}(y), 1\right), \min \left(p_{i} \circ I_{Q}^{U}(y), 0\right)\right]\right\}=\phi
$$

Complementation Properties 4.4 The Complementation properties of absolute $m$ IVF set $Y$ and null $m$ IVF set $\phi$ hold and given follows,

$$
\begin{gathered}
\phi^{c}=Y \text { and } \\
Y^{c}=\phi
\end{gathered}
$$

Double Complementation Property 4.5 Double complentation property holds for $m$ IVF set $Q$ over universal set $Y$

$$
\begin{gathered}
\left(Q^{c}\right)^{c}=\left\{y,\left([0,1]-p_{i} \circ I_{Q}(y)\right)\right\}^{c}=\left\{y,\left([0,1]-[0,1]+p_{i} \circ I_{Q}(y)\right)\right\}=Q \\
\text { for all } i=1,2, \cdots, m
\end{gathered}
$$

Exclusion and Contradiction Property 4.6 The Exclusion and Contradiction Property for $m$ IVF set $Q$ over universal set $Y$ holds and given as.

$$
\begin{aligned}
& Q \hat{\cup} Q^{c}=\left\{y,\left[p_{i} \circ I_{Q}^{L}(y), p_{i} \circ I_{Q}^{U}(y)\right]\right\} \hat{\cup}\left\{y,\left([0,1]-\left[p_{i} \circ I_{Q}^{L}(y), p_{i} \circ I_{Q}^{U}(y)\right]\right)\right\} \\
& =\left\{y,\left[p_{i} \circ I_{Q}^{L}(y), p_{i} \circ I_{Q}^{U}(y)\right]\right\} \hat{\cup}\left\{y,\left[0, p_{i} \circ I_{A}^{L}(y)\right) \cup\left(p_{i} \circ I_{A}^{U}(y), 1\right]\right\}=Y
\end{aligned}
$$

and

$$
\begin{gathered}
Q \hat{\cap} Q^{c}=\left\{y,\left[p_{i} \circ I_{Q}^{L}(y), p_{i} \circ I_{Q}^{U}(y)\right]\right\} \hat{\cap}\left\{y,\left([0,1]-\left[p_{i} \circ I_{Q}^{L}(y), p_{i} \circ I_{Q}^{U}(y)\right]\right)\right\} \\
=\left\{y,\left[p_{i} \circ I_{Q}^{L}(y), p_{i} \circ I_{Q}^{U}(y)\right]\right\} \hat{\cap}\left\{y,\left[0, p_{i} \circ I_{Q}^{L}(y)\right) \cap\left(p_{i} \circ I_{Q}^{U}(y), 1\right]=\phi\right. \\
\text { for all } i=1,2, \cdots, m
\end{gathered}
$$

Commutative Properties 4.7 Let $\mathrm{Q}$ and $\mathrm{R}$ be two $m \mathrm{IVF}$ then following holds true.

(1) $Q \hat{\cup} R=R \hat{\cup} Q$

(2) $Q \hat{\cap} R=R \hat{\cap} Q$

(1)Proof : -

$$
\begin{gathered}
\text { L.H.S }=Q \hat{\cup} R \\
=\left\{y,\left[\min \left(p_{i} \circ I_{Q}^{L}(y), p_{i} \circ I_{R}^{L}(y)\right), \max \left(p_{i} \circ I_{Q}^{U}(y), p_{i} \circ I_{R}^{U}(y)\right)\right]\right\} \\
=\left\{y,\left[\min \left(p_{i} \circ I_{R}^{L}(y), p_{i} \circ I_{Q}^{L}(y), \max \left(p_{i} \circ I_{R}^{U}(y), p_{i} \circ I_{Q}^{U}(y)\right)\right]\right\}\right. \\
=R \hat{U} Q=R . H . S \\
\text { for all } i=1,2, \cdots, m
\end{gathered}
$$


(2)Proof :-

$$
\begin{gathered}
\text { L.H.S }=Q \hat{\cap} R \\
=\left\{y,\left[\max \left(p_{i} \circ I_{Q}^{L}(y), p_{i} \circ I_{R}^{L}(y)\right), \min \left(p_{i} \circ I_{Q}^{U}(y), p_{i} \circ I_{R}^{U}(y)\right)\right]\right\} \\
=\left\{y,\left[\max \left(p_{i} \circ I_{R}^{L}(y), p_{i} \circ I_{Q}^{L}(y), \min \left(p_{i} \circ I_{R}^{U}(y), p_{i} \circ I_{Q}^{U}(y)\right)\right]\right\}\right. \\
=R \hat{\cap} Q=R . H . S \\
\text { for all } i=1,2, \cdots, m
\end{gathered}
$$

Associative Properties 4.8 Associative properties hold for $m$ IVF sets $Q, R$ and $S$ over universal set $Y$ as

$$
\begin{aligned}
& \text { (1) } Q \hat{\cup}(R \hat{\cup} S)=(Q \hat{\cup} R) \hat{\cup} S \\
& \text { (2) } Q \hat{\cap}(R \hat{\cap} S)=(Q \hat{\cap} R) \hat{\cap} S \\
& \text { (1)Proof : - } \\
& \text { L.H.S }=Q \hat{\cup}(R \hat{\cup} S) \\
& =Q \hat{\cup}\left\{y,\left[\min \left(p_{i} \circ I_{R}^{L}(y), p_{i} \circ I_{S}^{L}(y)\right), \max \left(p_{i} \circ I_{R}^{U}(y), p_{i} \circ I_{S}^{U}(y)\right)\right]\right\} \\
& =\left\{y,\left[\min \left(p_{i} \circ I_{Q}^{L}(y), p_{i} \circ I_{R}^{L}(y), p_{i} \circ I_{S}^{L}(y)\right), \max \left(p_{i} \circ I_{Q}^{U}(y), p_{i} \circ I_{R}^{U}(y), p_{i} \circ I_{S}^{U}(y)\right)\right]\right\} \\
& =\left\{y,\left[\left(\min \left(p_{i} \circ I_{Q}^{L}(y), p_{i} \circ I_{R}^{L}(y)\right), \max \left(p_{i} \circ I_{Q}^{U}(y), p_{i} \circ I_{R}^{U}(y)\right)\right]\right\} \hat{\cup} S\right. \\
& =(Q \hat{\cup} R) \hat{\cup} S=R . H . S \\
& \text { (2)Proof :- } \\
& \text { for all } i=1,2, \cdots, m \\
& L . H . S=Q \hat{\cap}(R \hat{\cap} S) \\
& =Q \hat{\cap}\left\{y,\left[\max \left(p_{i} \circ I_{R}^{L}(y), p_{i} \circ I_{S}^{L}(y)\right), \min \left(p_{i} \circ I_{R}^{U}(y), p_{i} \circ I_{S}^{U}(y)\right)\right]\right\} \\
& =\left\{y,\left[\max \left(p_{i} \circ I_{Q}^{L}(y), p_{i} \circ I_{R}^{L}(y), p_{i} \circ I_{S}^{L}(y)\right), \min \left(p_{i} \circ I_{Q}^{U}(y), p_{i} \circ I_{R}^{U}(y), p_{i} \circ I_{S}^{U}(y)\right)\right]\right\} \\
& =\left\{y,\left[\left(\max \left(p_{i} \circ I_{Q}^{L}(y), p_{i} \circ I_{R}^{L}(y)\right), \min \left(p_{i} \circ I_{Q}^{U}(y), p_{i} \circ I_{R}^{U}(y)\right)\right]\right\} \cap \hat{\cap} S\right. \\
& =(Q \hat{\cap} R) \hat{\cap} S=R . H . S \\
& \text { for all } i=1,2, \cdots, m
\end{aligned}
$$

Distributive Properties 4.9 Distributive properties hold for $m \mathrm{IVF}$ sets $Q, R$ and $S$ over universal set $Y$ as

(1) $Q \hat{\cup}(R \hat{\cap} S)=(Q \hat{\cup} R) \hat{\cap}(Q \hat{\cup} S)$

(2) $Q \hat{\cap}(R \hat{\cup} S)=(Q \hat{\cap} R) \hat{\cup}(Q \hat{\cap} S)$

(1)Proof :-

$$
\begin{gathered}
R . H . S=(Q \hat{\cup} R) \hat{\cap}(Q \hat{\cup} S) \\
=\left\{y,\left[\min \left(p_{i} \circ I_{Q}^{L}(y), p_{i} \circ I_{R}^{L}(y)\right), \max \left(p_{i} \circ I_{Q}^{U}(y), p_{i} \circ I_{R}^{U}(y)\right)\right]\right\} \\
\hat{\cap}\left\{y,\left[\min \left(p_{i} \circ I_{Q}^{L}(y), p_{i} \circ I_{S}^{L}(y)\right), \max \left(p_{i} \circ I_{Q}^{U}(y), p_{i} \circ I_{S}^{U}(y)\right)\right]\right\} \\
=\left\{y,\left[\max \left(\min \left(p_{i} \circ I_{Q}^{L}(y), p_{i} \circ I_{R}^{L}(y)\right), \min \left(p_{i} \circ I_{Q}^{L}(y), p_{i} \circ I_{S}^{L}(y)\right)\right),\right.\right. \\
\left.\left.\min \left(\max \left(p_{i} \circ I_{Q}^{U}(y), p_{i} \circ I_{R}^{U}(y)\right), \max \left(p_{i} \circ I_{Q}^{U}(y), p_{i} \circ I_{S}^{U}(y)\right)\right)\right]\right\} \\
=\left\{y,\left[\min \left(p_{i} \circ I_{Q}^{L}(y), \max \left(p_{i} \circ I_{R}^{L}(y), p_{i} \circ I_{S}^{L}(y)\right)\right),\right.\right. \\
\left.\left.\max \left(p_{i} \circ I_{Q}^{U}(y), \min \left(p_{i} \circ I_{R}^{U}(y), p_{i} \circ I_{S}^{U}(y)\right)\right)\right]\right\} \\
= \\
= \\
\left\{y,\left[p_{i} \circ I_{Q}^{L}(y), p_{i} \circ I_{Q}^{U}(y)\right]\right\} \hat{\cup}\left\{y,\left[\max \left(p_{i} \circ I_{R}^{L}(y), p_{i} \circ I_{S}^{L}(y)\right), \min \left(p_{i} \circ I_{R}^{U}(y), p_{i} \circ I_{S}^{U}(y)\right)\right]\right\} \\
=Q \hat{\cup}(R \hat{\cap} S)=L . H . S \\
\text { for all } i=1,2, \cdots, m
\end{gathered}
$$


(2)Proof : -

$$
\begin{gathered}
R . H . S=(Q \hat{\cap} R) \hat{\cup}(Q \hat{\cap} S) \\
=\left\{y,\left[\max \left(p_{i} \circ I_{Q}^{L}(y), p_{i} \circ I_{R}^{L}(y)\right), \min \left(p_{i} \circ I_{Q}^{U}(y), p_{i} \circ I_{R}^{U}(y)\right)\right]\right\} \\
\hat{\cup}\left\{y,\left[\max \left(p_{i} \circ I_{Q}^{L}(y), p_{i} \circ I_{S}^{L}(y)\right), \min \left(p_{i} \circ I_{Q}^{U}(y), p_{i} \circ I_{S}^{U}(y)\right)\right]\right\} \\
=\left\{y,\left[\min \left(\max \left(p_{i} \circ I_{Q}^{L}(y), p_{i} \circ I_{R}^{L}(y)\right), \max \left(p_{i} \circ I_{Q}^{L}(y), p_{i} \circ I_{S}^{L}(y)\right)\right),\right.\right. \\
\left.\left.\max \left(\min \left(p_{i} \circ I_{Q}^{U}(y), p_{i} \circ I_{R}^{U}(y)\right), \min \left(p_{i} \circ I_{Q}^{U}(y), p_{i} \circ I_{S}^{U}(y)\right)\right)\right]\right\} \\
=\left\{y,\left[\max \left(p_{i} \circ I_{Q}^{L}(y), \min \left(p_{i} \circ I_{R}^{L}(y), p_{i} \circ I_{S}^{L}(y)\right)\right),\right.\right. \\
\left.\left.\min \left(p_{i} \circ I_{Q}^{U}(y), \max \left(p_{i} \circ I_{R}^{U}(y), p_{i} \circ I_{S}^{U}(y)\right)\right)\right]\right\}
\end{gathered}
$$

$\left\{y,\left[p_{i} \circ I_{Q}^{L}(y), p_{i} \circ I_{Q}^{U}(y)\right]\right\} \hat{\cap}\left\{y,\left[\min \left(p_{i} \circ I_{R}^{L}(y), p_{i} \circ I_{S}^{L}(y)\right), \max \left(p_{i} \circ I_{R}^{U}(y), p_{i} \circ I_{S}^{U}(y)\right)\right]\right\}$

$$
=Q \hat{\cap}(R \hat{\cup} S)=L . H . S
$$

$$
\text { for all } i=1,2, \cdots, m
$$

De Morgan's Laws 4.10 De Morgan's laws for $m$ IVF sets are given as follows

(1) $(Q \hat{\cup} R)^{c}=Q^{c} \hat{\cap} R^{c}$

(2) $(Q \hat{\cap} R)^{c}=Q^{c} \hat{\cup} R^{c}$

(1)Proof : -

$$
\begin{gathered}
\text { R.H.S }=Q^{c} \hat{\cap} R^{c} \\
=\left\{y,[0,1]-\left[p_{i} \circ I_{Q}^{L}(y), p_{i} \circ I_{Q}^{U}(y)\right]\right\} \hat{\cap}\left\{y,[0,1]-\left[p_{i} \circ I_{R}^{L}(y), p_{i} \circ I_{R}^{U}(y)\right]\right\} \\
=\left\{y,\left[0, p_{i} \circ I_{Q}^{L}(y)\right) \cup\left(p_{i} \circ I_{Q}^{U}(y), 1\right]\right\} \hat{\cap}\left\{y,\left[0, p_{i} \circ I_{R}^{L}(y)\right) \cup\left(p_{i} \circ I_{R}^{U}(y), 1\right]\right\} \\
=\left\{y,\left[\max (0,0), \min \left(p_{i} \circ I_{Q}^{L}(y), p_{i} \circ I_{R}^{L}(y)\right)\right)\right\} \\
\cup\left\{y,\left(\max \left(p_{i} \circ I_{Q}^{U}(y), p_{i} \circ I_{R}^{U}(y)\right), \min (1,1)\right]\right\} \\
=\left\{y,\left[0, \min \left(p_{i} \circ I_{Q}^{L}(y), p_{i} \circ I_{R}^{L}(y)\right) \cup\left(\max \left(p_{i} \circ I_{Q}^{U}(y), p_{i} \circ I_{R}^{U}(y)\right), 1\right]\right\}\right. \\
=\left\{y,[0,1]-\left[\min \left(p_{i} \circ I_{Q}^{L}(y), p_{i} \circ I_{R}^{L}(y)\right), \max \left(p_{i} \circ I_{Q}^{U}(y), p_{i} \circ I_{Q}^{U}(y)\right)\right]\right\} \\
=(Q \cup R)^{c} \\
=L . H . S \\
\text { for all } i=1,2, \cdots, m
\end{gathered}
$$

(2)Proof : -

$$
\begin{gathered}
\text { R.H.S }=Q^{c} \hat{\cup} R^{c} \\
=\left\{y,[0,1]-\left[p_{i} \circ I_{Q}^{L}(y), p_{i} \circ I_{Q}^{U}(y)\right]\right\} \hat{\cup}\left\{y,[0,1]-\left[p_{i} \circ I_{R}^{L}(y), p_{i} \circ I_{R}^{U}(y)\right]\right\} \\
=\left\{y,\left[0, p_{i} \circ I_{Q}^{L}(y)\right) \cup\left(p_{i} \circ I_{Q}^{U}(y), 1\right]\right\} \hat{\cup}\left\{y,\left[0, p_{i} \circ I_{R}^{L}(y)\right) \cup\left(p_{i} \circ I_{R}^{U}(y), 1\right]\right\} \\
=\left\{y,\left[\min (0,0), \max \left(p_{i} \circ I_{Q}^{L}(y), p_{i} \circ I_{R}^{L}(y)\right)\right)\right\} \\
\cup\left\{y,\left(\min \left(p_{i} \circ I_{Q}^{U}(y), p_{i} \circ I_{R}^{U}(y)\right), \max (1,1)\right]\right\} \\
=\left\{y,\left[0, \max \left(p_{i} \circ I_{Q}^{L}(y), p_{i} \circ I_{R}^{L}(y)\right) \cup\left(\min \left(p_{i} \circ I_{Q}^{U}(y), p_{i} \circ I_{R}^{U}(y)\right), 1\right]\right\}\right. \\
=\left\{y,[0,1]-\left[\max \left(p_{i} \circ I_{Q}^{L}(y), p_{i} \circ I_{R}^{L}(y)\right), \min \left(p_{i} \circ I_{Q}^{U}(y), p_{i} \circ I_{Q}^{U}(y)\right)\right]\right\} \\
=(Q \hat{\cap} R)^{c} \\
=L . H . S \\
\text { for all } i=1,2, \cdots, m
\end{gathered}
$$




\section{Distance and Similarity Measure}

Definition 5.1 (Distances of $m \mathrm{IVF}$ sets). Let $Q$ and $R$ be two $m I V F$ sets over $Y=\left\{y_{1}, y_{2}, \cdots, y_{n}\right\}$ defined as.

$$
\begin{gathered}
Q=\left\{y_{j}, p_{i} \circ I_{Q}\left(y_{j}\right)\right\} \\
R=\left\{y_{j}, p_{i} \circ I_{R}\left(y_{j}\right)\right\} \\
\text { for all } i=1,2, \cdots, m \text { and } j=1,2, \cdots, n
\end{gathered}
$$

where,

$$
\begin{gathered}
p_{i} \circ I_{Q}\left(y_{j}\right)=\left[p_{i} \circ I_{Q}^{L}\left(y_{j}\right), p_{i} \circ I_{Q}^{U}\left(y_{j}\right)\right], \\
p_{i} \circ I_{R}\left(y_{j}\right)=\left[p_{i} \circ I_{R}^{L}\left(y_{j}\right), p_{i} \circ I_{R}^{U}\left(y_{j}\right)\right] \\
\quad \text { for all } i=1,2, \cdots, m \text { and } j=1,2, \cdots, n
\end{gathered}
$$

Their distances will defined as

Hamming distance

$$
\operatorname{dis}_{H}(Q, R)=\frac{1}{m}\left\{\sum_{i=1}^{m} \sum_{j=1}^{n}\left|p_{i} \circ \alpha_{j}-p_{i} \circ \beta_{j}\right|\right\}
$$

\section{Normalized Hamming distance}

$$
\operatorname{dis}_{N H}(Q, R)=\frac{1}{m n}\left\{\sum_{i=1}^{m} \sum_{j=1}^{n}\left|p_{i} \circ \alpha_{j}-p_{i} \circ \beta_{j}\right|\right\}
$$

\section{Euclidean distance}

$$
\operatorname{dis}_{E}(Q, R)=\sqrt{\frac{1}{m}\left\{\sum_{i=1}^{m} \sum_{j=1}^{n}\left(p_{i} \circ \alpha_{j}-p_{i} \circ \beta_{j}\right)^{2}\right\}}
$$

\section{Normalized Euclidean distance}

$$
\operatorname{dis}_{N E}(Q, R)=\sqrt{\frac{1}{m n}\left\{\sum_{i=1}^{m} \sum_{j=1}^{n}\left(p_{i} \circ \alpha_{j}-p_{i} \circ \beta_{j}\right)^{2}\right\}}
$$

where,

$$
p_{i} \circ \alpha_{j}=\frac{p_{i} \circ I_{Q}^{L}\left(y_{j}\right)+p_{i} \circ I_{Q}^{U}\left(y_{j}\right)}{2}
$$

and

$$
p_{i} \circ \beta_{j}=\frac{p_{i} \circ I_{R}^{L}\left(y_{j}\right)+p_{i} \circ I_{R}^{U}\left(y_{j}\right)}{2}
$$

Theorem 5.2. The distance measure between mIVF sets $Q$ and $R$ satisfy the following inequalities.
(1) $\operatorname{dis}_{H}(Q, R) \leq n$,
$(2) \operatorname{dis}_{N H}(Q, R) \leq 1$, 
$(3) \operatorname{dis}_{E}(Q, R) \leq \sqrt{n}$

(4) $\operatorname{dis}_{N E}(Q, R) \leq 1$,

Theorem 5.3. The distance functions $d i s_{H}$, dis ${ }_{N H}$, dis ${ }_{E}$ and dis $s_{N E}$ defined from $m I V F^{Y} \rightarrow R^{+}$, are metric distances.

Proof. Let $Q, R$ and $S$ be three mIVF sets over universal set $Y$, then

(1) $\operatorname{dis}_{H}(Q, R) \geq 0$

(2) Suppose $\operatorname{dis}_{H}(Q, R)=0$

$$
\Leftrightarrow \frac{1}{m}\left\{\sum_{i=1}^{m} \sum_{j=1}^{n}\left|p_{i} \circ \alpha_{j}-p_{i} \circ \beta_{j}\right|\right\}=0
$$

for all $i, j, k$

$$
\begin{gathered}
\Leftrightarrow\left|p_{i} \circ \alpha_{j}-p_{i} \circ \beta_{j}\right|=0 \\
\Leftrightarrow p_{i} \circ \alpha_{j}=p_{i} \circ \beta_{j} \\
\Leftrightarrow Q=R
\end{gathered}
$$

(3) $\operatorname{dis}_{H}(Q, R)=\operatorname{dis}_{H}(R, Q)$

(4) For any three mIVF sets $Q, R$ and $S$,

$$
\begin{gathered}
\left|p_{i} \circ \alpha_{j}-p_{i} \circ \beta_{j}\right| \\
=\left|p_{i} \circ \alpha_{j}-p_{i} \circ \gamma_{j}+p_{i} \circ \gamma_{j}-p_{i} \circ \beta_{j}\right| \\
\leq\left|p_{i} \circ \alpha_{j}-p_{i} \circ \gamma_{j}\right|+\left|p_{i} \circ \gamma_{j}-p_{i} \circ \beta_{j}\right|
\end{gathered}
$$

Thus,

$$
\operatorname{dis}_{H}(Q, R) \leq d i s_{H}(Q, S)+\operatorname{dis}_{H}(S, R)
$$

Definition 5.4 (Similarity Measure). [6]

The Similarity Measure(Sim.M) of two mIVF set $Q$ and $R$ can be defined as

$$
\operatorname{Sim} . M(Q, R)=\frac{1}{1+\operatorname{dis}(Q, R)}
$$

Definition 5.5 (Similarity). [6] Two mIVF sets $Q$ and $R$ are $\sigma$ similar if and only if $\operatorname{Sim.M}(Q, R) \geq \sigma$, i.e.

$$
Q \approx^{\sigma} R \Leftrightarrow \operatorname{Sim} \cdot M(Q, R) \geq \sigma, \sigma \in(0,1)
$$

$Q$ and $R$ are significantly similar if $\operatorname{Sim} . M(Q, R) \geq \frac{1}{2}$.

Example 5.6. If $Q$ and $R$ be two mIVF set over $Y=\left\{y_{1}, y_{2}\right\}$ such that

$$
\begin{aligned}
& Q=\left\{\left(y_{1},[0.22,0.35],[0.40,0.53]\right),\left(y_{2},[0.32,0.36],[0.44,0.54]\right)\right\} \\
& R=\left\{\left(y_{1},[0.37,0.69],[0.23,0.53]\right),\left(y_{2},[0.10,0.80],[0.22,0.24]\right)\right\}
\end{aligned}
$$

then Hamming distance is

$$
\operatorname{dis}_{H}(Q, R)=0.405
$$

and similarity measure will be 


$$
\operatorname{Sim} \cdot M(Q, R)=\frac{1}{1+0.405}=0.7117
$$

It shows Set $Q$ is significantly similar to set $R$.

Theorem 5.7. The Sim.M of two mIVF sets $Q$ and $R$ satisfies the following.

(1). $0 \leq \operatorname{Sim} \cdot M(Q, R) \leq 1$

(2). $\operatorname{Sim} \cdot M(Q, R)=\operatorname{Sim} \cdot M(R, Q)$

(3). If $\operatorname{Sim} \cdot M(Q, R)=1 \Leftrightarrow Q=R$

5.8. A Numerical Example. In this section, an algorithm is given to compute similarity measure for the structure of $m I V F$ to the application of pattern/brand recognition. Proposed algorithm can be applicable in any field of the patten recognition where more than one opinions are given to single attributive value in the form of interval-valued fuzzy membership.

\section{Algorithm}

Step 1 : Assume that there are $n$ number of watches, which are represented by mIVF set $H_{k}, k=1,2,3, \cdots n$, in feature space $E T$.

Step 2 : Consider an $m$ IVF Set $\varrho$ is unknown brand watch, that is needed to be recognized. Step 3 : Convert the interval-valued membership values into multipolar $\mathrm{mF}$ set, by using the formula

$$
p_{i} \circ \alpha_{H k j}=\frac{p_{i} \circ I_{H_{k}}^{L}\left(y_{j}\right)+p_{i} \circ I_{H_{k}}^{U}\left(y_{j}\right)}{2}
$$

and

$$
p_{i} \circ \beta_{j}=\frac{p_{i} \circ I_{\varrho}^{L}\left(y_{j}\right)+p_{i} \circ I_{\varrho}^{U}\left(y_{j}\right)}{2}
$$

which changes $I_{H_{k}}\left(y_{j}\right)$ and $I_{\varrho}\left(y_{j}\right)$ the interval-valued data into $H_{k}$ and $\varrho$ the $\mathrm{mF}$ set. Step 4 : After that calculate $d i s_{H}$, dis $s_{N H}, d i s_{E}$, dis $s_{N}$ distance between $H_{k}$ and $\varrho$ Step 5 : Calculate $\operatorname{Sim} . M\left(H_{k}, \varrho\right)$ between $H_{k}$ and $\varrho$, using the formula

$$
\operatorname{Sim} . M\left(H_{k}, \varrho\right)=\frac{1}{1+\operatorname{dis}\left(H_{k}, \varrho\right)}
$$

Step 6 : Lastly, analyze the findings by selecting the Hk, which has the greatest value of similarity measure with unknown brand watch $\varrho$.

Assume that there are four brands of watches denoted by $H_{1}, H_{2}, H_{3}$ and $H_{4}$. Let $E T=$ $\left\{e t_{1}=\right.$ Material, $e t_{2}=$ Glass kind, $e t_{3}=$ Water Resistance, et $_{4}=$ Beautiful Finish $\}$ be feature space of watches. We construct Four 2-IVF sets (shown in Table 1) and also construct $2-$ IVF set of unknown watch as $\varrho$,

$$
\begin{gathered}
\varrho=\left\{\left(e_{1},[0.37,0.4][0.92,0.5]\right),\left(e_{2},[0.71,0.5],[0.41,0.73]\right),\right. \\
\left.\left(e_{3},[0.93,0.95][0.62,0.22]\right),\left(\text { et }_{4}[0.13,0.51][0.11,0.73]\right)\right\}
\end{gathered}
$$

Then we convert 2 -IVF sets $H_{1}, H_{2}, H_{3}, H_{4}$ set into $2-\mathrm{F}$ sets (shown in Table 2 ) and $\varrho$ into $2-\mathrm{F}$ sets as follows using equation (5.5) and (5.6).

$$
\begin{aligned}
\varrho=\left\{\left(e t_{1},(0.385,0.915)\right),\left(e t_{2},(0.605,0.57)\right),\right. \\
\left.\left(e t_{3},(0.94,0.42)\right),\left(e t_{4}(0.32,0.42)\right)\right\}
\end{aligned}
$$




\begin{tabular}{ccccc}
\hline brands & $e t_{1}$ & $e t_{2}$ & $e t_{3}$ & et $_{4}$ \\
\hline$H_{1}$ & {$[0.20,0.35],[0.40,0.50]$} & {$[0.30,0.60],[0.40 .0 .35]$} & {$[0.10,0.71],[0.92,0.50]$} & {$[0.71,0.92],[0.24,0.39]$} \\
$H_{2}$ & {$[0.20,0.40],[0.40,0.50]$} & {$[0.30,0.50],[0.20 .0 .50]$} & {$[0.30,0.50],[0.10,0.40]$} & {$[0.17,0.95],[0.21,0.40]$} \\
$H_{3}$ & {$[0.30,0.50],[0.40,0.50]$} & {$[0.30,0.80],[0.40 .0 .90]$} & {$[0.40,0.70],[0.10,0.20]$} & {$[0.30,0.90],[0.40,0.20]$} \\
$H_{4}$ & {$[0.71,0.11],[0.30,0.40]$} & {$[0.12,0.90],[0.30 .0 .92]$} & {$[0.15,0.40],[0.50,0.70]$} & {$[0.31,0.57],[0.41,0.72]$} \\
\hline
\end{tabular}

TABLE 1 . Represents four $2-$ IVF sets $H_{1}, H_{2}, H_{3}, H_{4}$

\begin{tabular}{ccccc}
\hline brands & $e t_{1}$ & $e t_{2}$ & $e t_{3}$ & $e t_{4}$ \\
\hline$H_{1}$ & $(0.275,0.45)$ & $(0.45,0.375)$ & $(0.405,0.71)$ & $(0.815,0.315)$ \\
$H_{2}$ & $(0.30,0.45)$ & $(0.40,0.35)$ & $(0.40,0.25)$ & $(0.56,0.34)$ \\
$H_{3}$ & $(0.40,0.45)$ & $(0.55,0.65)$ & $(0.55,0.15)$ & $(0.60,0.30)$ \\
$H_{4}$ & $(0.41,0.35)$ & $(0.51,0.61)$ & $(0.275,0.60)$ & $(0.44,0.565)$ \\
\hline
\end{tabular}

TABLE 2. Represents four $2-$ polar Fuzzy sets $H_{1}, H_{2}, H_{3}, H_{4}$

Euclidean distance formula (5.3) gives the distance measure of $H_{k}$ and $\varrho$ as

$$
\begin{aligned}
& d_{E}\left(H_{1}, \varrho\right)=0.6968 \\
& d_{E}\left(H_{2}, \varrho\right)=0.6185 \\
& d_{E}\left(H_{3}, \varrho\right)=0.5352 \\
& d_{E}\left(H_{4}, \varrho\right)=0.6632
\end{aligned}
$$

By using Similarity Measure formula (5.7) (Sim.M) is

$$
\begin{aligned}
& \operatorname{Sim} . M\left(H_{1}, \varrho\right)=\frac{1}{1+d_{E}\left(H_{1}, \varrho\right)}=\frac{1}{1+0.6968}=0.5893 \\
& \operatorname{Sim} \cdot M\left(H_{2}, \varrho\right)=\frac{1}{1+d_{E}\left(H_{2}, \varrho\right)}=\frac{1}{1+0.6185}=0.6178 \\
& \operatorname{Sim} \cdot M\left(H_{3}, \varrho\right)=\frac{1}{1+d_{E}\left(H_{3}, \varrho\right)}=\frac{1}{1+0.5352}=0.6513 \\
& \operatorname{Sim} \cdot M\left(H_{4}, \varrho\right)=\frac{1}{1+d_{E}\left(H_{4}, \varrho\right)}=\frac{1}{1+0.6632}=0.6012
\end{aligned}
$$

While Sim.M is greater than 0.5 for all brands, it is $H_{3}$ that is much greater than the others, which is why the unknown watch varrho is too near to brand $H_{3}$.

5.9. Limitation of the Method. There are several limitations of the method that must be assured before implementing the similarity measure criteria.

(1) Similarity measure can be found between two sets at a time to find comparison among themselves.

(2) The two sets must be independent of each other and must be from the same structure. 


\section{TOPSIS}

A methodology to extend the TOPSIS to m-polar interval-valued set is determined in this section. This process is very applicable to deal with the group decision-making problem under $m$ IVF system.

Each criterion's concern weight can be determined either directly or indirectly by pairwise comparisons. Suppose that there is a group of $m$ decision-makers who assess the scores of alternatives $\ddot{A}_{j}(j=1,2, \operatorname{cdots}, p)$ based on criteria $\ddot{C}_{k}(k=1,2, \operatorname{cdots}, q)$ in interval-valued membership, while the evaluation of criterion weights is perceived. A matrix representation of a multi-criteria multi-person decision-making problem is as fol- lows:

$$
\ddot{G}=\left[\begin{array}{cccc}
g_{11}^{I} & g_{12}^{I} & \cdots & g_{1 q}^{I} \\
g_{21}^{I} & g_{22}^{I} & \cdots & g_{2 q}^{I} \\
\vdots & \vdots & \ddots & \vdots \\
g_{p 1}^{I} & g_{p 2}^{I} & \cdots & g_{p q}^{I}
\end{array}\right]_{p \times q}
$$

where $p$ denotes the number of alternatives, $q$ denotes the number of criteria and $g_{j k}^{I}$ for all $j, k$; represents the ratings of $j t h$-alternatives concering the $k t h$-criteria in the intervalvalued. For ranting $g_{j k}^{I}$, the data of $\mathrm{m}$ decision-makers can be represented as

$$
\begin{gathered}
\qquad g_{j k}^{I}=\left[p_{i} \circ g_{j k}^{L}, p_{i} \circ g_{j k}^{U}\right] \\
\text { where } i=1,2, \cdots, m ; j=1,2, \cdots, p \text {; and } k=1,2, \cdots, q
\end{gathered}
$$

Convert the given multipolar interval valued membership evaluation of alternatives into multipolar $m$-single-valued membership evaluation by,

$$
\begin{gathered}
p_{i} \circ g_{j k}=\frac{p_{i} \circ g_{j k}^{L}+p_{i} \circ g_{j k}^{U}}{2} \\
\text { for all } i=1,2, \cdots, m
\end{gathered}
$$

then we get rating of alternatives as,

$$
\begin{gathered}
\ddot{g}_{j k}=\left(p_{1} \circ g_{j k}, p_{2} \circ g_{j k}, \cdots, p_{m} \circ g_{j k}\right) \\
\quad \text { where } j=1,2, \cdots, p ; \text { and } k=1,2, \cdots, q
\end{gathered}
$$

and the criteria weights are presented in multipolar information as

$$
\begin{gathered}
\ddot{w}_{k}=\left(p_{1} \circ w_{k}, p_{2} \circ w_{k}, \cdots, p_{m} \circ w_{k}\right) \\
\text { where } k=1,2, \cdots, q
\end{gathered}
$$

As fuzzy numbers belong to $[0,1]$, then by normalization we get the normalized $m \mathrm{~F}$ decision matrix denoted as $\ddot{R}$ and

$$
\begin{gathered}
\ddot{R}=\left[\ddot{r}_{j k}\right]_{p \times q} \text { where, } \\
\ddot{r}_{j k}=\left(\begin{array}{c}
\left.p_{i} \circ r_{j k}=\frac{p_{i} \circ g_{j k}}{\sqrt{\sum_{j=1}^{p}\left(p_{i} \circ g_{j k}\right)^{2}}}\right) \\
\text { for all } i=1,2, \cdots, m
\end{array}\right.
\end{gathered}
$$

The above-mentioned normalisation method preserves the property of the mIVF set that the ranges of membership of elements are $[0,1]$. We establish the weighted normalised $m$ IVF decision matrix, denoted as $\ddot{V}$, by taking into account the various importance of each criterion, 


$$
\begin{gathered}
\ddot{V}=\left[\ddot{v}_{j k}\right]_{p \times q} \text { where, } \\
\ddot{v}_{j k}=\ddot{r}_{j k}(\cdot) \ddot{w}_{k}=\left(p_{i} \circ r_{j k}(\cdot) p_{i} \circ w_{k}\right) \\
\text { for all } i=1,2, \cdots, m
\end{gathered}
$$

In accordance with the weighted normalized $m \mathrm{~F}$ decision matrix, it can be seen that the elements $\ddot{v}_{j k}$ for all $i, j$; are normalized. Then, we evaluate the $m \mathrm{~F}$ positive-ideal solution $\left(m\right.$ FPIS, $\left.A^{*}\right)$ and $m$ F negative-ideal solution ( $m$ FNIS, $\left.A^{-}\right)$as

$$
\begin{gathered}
A^{*}=\left(\ddot{v}_{1}^{*}, \ddot{v}_{2}^{*}, \cdots, \ddot{v}_{q}^{*}\right), \\
A^{-}=\left(\ddot{v}_{1}^{-}, \ddot{v}_{2}^{-}, \cdots, \ddot{v}_{q}^{-}\right),
\end{gathered}
$$

where,

and

$$
\ddot{v}_{k}^{*}=\left(p_{i} \circ v_{k}^{*}\right)=\left\{\begin{array}{cc}
(1,1, \cdots, 1) & k \in B \\
(0,0, \cdots, 0) & k \in C .
\end{array}\right.
$$

$$
\begin{gathered}
\ddot{v}_{k}^{-}=\left(p_{i} \circ v_{k}^{-}\right)=\left\{\begin{array}{cc}
(0,0, \cdots, 0) & k \in B ; \\
(1,1, \cdots, 1) & k \in C .
\end{array}\right. \\
\text { for all } i=1,2, \cdots, m \text { and } k=1,2, \cdots, q
\end{gathered}
$$

where $B$ and $C$ denotes the benefit and cost criteria respectively. The Euclidean distance (Separation) of each alternative from $A^{*}$ and $A^{-}$can be calculated as

$$
\begin{gathered}
S_{j}^{*}=d\left(\ddot{v}_{j k}, \ddot{v}_{k}^{*}\right)=\sqrt{\frac{1}{m}\left\{\sum_{i=1}^{m} \sum_{k=1}^{q}\left(p_{i} \circ v_{j k}-p_{i} \circ v_{k}^{*}\right)^{2}\right\}} \\
S_{j}^{-}=d\left(\ddot{v}_{j k}, \ddot{v}_{k}^{-}\right)=\sqrt{\frac{1}{m}\left\{\sum_{i=1}^{m} \sum_{k=1}^{q}\left(p_{i} \circ v_{j k}-p_{i} \circ v_{k}^{-}\right)^{2}\right\}}
\end{gathered}
$$

Next move forward to the closeness coefficient to rank all alternatives after computation of $S_{j}^{*}$ and $S_{j}^{-}$of each alternative $\ddot{A}_{j},(j=1,2, \cdots, p)$.

$$
C . C o f_{j}=\frac{S_{j}^{-}}{S_{j}^{-}+S_{j}^{*}}
$$

As the closeness coefficient $C . C o f_{j}$ approaches one, the alternative $\ddot{A}_{j}$ is similar to $m$ FPIS $\left(A^{*}\right)$ and far from $m$ FNIS $\left(A^{-}\right)$. As a result, using the $C . C o f_{j}$, we will rate all alternatives and choose the best one that is closest to 1 .

\section{Algorithm}

In short, the algorithm of the multi-decision maker multi-criteria decision-making in the approach of $m$ IVF set is given as follows.

Step 1: Make a group of decision-makers, then analyzes the evaluation criteria.

Step 2: Evaluate the ratings of alternatives $\ddot{A}_{j}$ concerning criteria $\ddot{C}_{k}$ in interval-valued and weight of criterion.

Step 3: Create an $m \mathrm{IV}$ decision matrix.

Step 4: Convert the $m$ IV data into $m$-single-valued.

Step 5: Normalized $m \mathrm{SV}$ to get $m \mathrm{~F}$ decision matrix.

Step 6: Create the weighted normalized $m \mathrm{~F}$ decision matrix. 


\begin{tabular}{ccccc}
\hline & $\ddot{C}_{1}$ & $\ddot{C}_{2}$ & $\ddot{C}_{3}$ & $\ddot{C}_{4}$ \\
\hline$\ddot{A}_{1}$ & $([3,5],[2,5],[1,4])$ & $([5,6],[5,5],[5,7])$ & $([4,7],[4,6],[6,7])$ & $([7,9],[8,10],[6,9])$ \\
$\ddot{A}_{2}$ & $([6,8],[5,7],[8,8])$ & $([6,6],[5,7],[4,7])$ & $([2,2],[2,4],[1,2])$ & $([1,3],[2,6],[3,5])$ \\
$\ddot{A}_{3}$ & $([6,7],[8,9],[9,10])$ & $([5,7],[3,6],[4,4])$ & $([4,6],[5,8],[4,6])$ & $([6,7],[3,6],[4,8])$ \\
$\ddot{A}_{4}$ & $([2,2],[3,4],[1,3])$ & $([6,8],[6,9],[6,7])$ & $([9,9],[8,10],[9,9])$ & $([2,6],[3,7],[3,6])$ \\
$\ddot{w}$ & $(0.32,0.25,0.28)$ & $(0.24,0.26,0.26)$ & $(0.21,0.29,0.23)$ & $(0.23,0.20,0.23)$ \\
\hline
\end{tabular}

TABLE 3. Represents 3-IV decision matrix and 3-polar weights

\begin{tabular}{ccccc}
\hline & $\ddot{C}_{1}$ & $\ddot{C}_{2}$ & $\ddot{C}_{3}$ & $\ddot{C}_{4}$ \\
\hline$\ddot{A}_{1}$ & $(4,3.5,2.5)$ & $(5.5,5,6)$ & $(5.5,5,6.5)$ & $(8,9,7.5)$ \\
$\ddot{A}_{2}$ & $(7,6,8)$ & $(6,6,5.5)$ & $(2,3,1.5)$ & $(2,4,4)$ \\
$\ddot{A}_{3}$ & $(6.5,8.5,9.5)$ & $(6,4.5,4)$ & $(5,6.5,5)$ & $(6.5,4.5,6)$ \\
$\ddot{A}_{4}$ & $(2,3.5,2)$ & $(7,7.5,6.5)$ & $(9,9,9)$ & $(4,5,4.5)$ \\
\hline \multicolumn{5}{c}{ TABLE 4. 3-polar decision matrix }
\end{tabular}

Step 7: Determine the fuzzy positive ideal solution ( $m$ FPIS) and negative ideal solution ( $m$ FNIS).

Step 8: Compute the Separation measure of each alternative from $m$ FPIS and $m$ FNIS, respectively.

Step 9: Compute the closeness coefficient $C . C o f_{j}$ of each alternative.

Step 10: According to the closeness coefficient, give the rank to all alternatives and select the best one.

6.1. A Numerical Example. Assume an investment firm wants to do some investment in best alternative. The company established a committee of three individuals to choose the best option from a list of four potential alternatives for investing the money, $\ddot{A}_{1}$ is a telecommunication company $\ddot{A}_{2}$ is a food company $\ddot{A}_{3}$ is an electronics company $\ddot{A}_{4}$ is a medicine company

The committee must take following criteria under consideration for the decision making process

$\ddot{C}_{1}$ is the environmental impact analysis

$\ddot{C}_{2}$ is the risk analysis

$\ddot{C}_{3}$ is the social-political impact analysis

$\ddot{C}_{4}$ is the growth analysis

The committee of three decision-makers $p_{i}(i=1,2,3)$ evaluate all four possible alternatives using interval-valued data in the range $[0,10]$ and weight of criterion in fuzzy singlevalued as shown in Table 3.

Now, converting interval-valued data in above table to single-valued data as shown in Table 4 by using equation (6.9). 


\begin{tabular}{ccccc}
\hline & $\ddot{C}_{1}$ & $\ddot{C}_{2}$ & $\ddot{C}_{3}$ & $\ddot{C}_{4}$ \\
\hline$\ddot{A}_{1}$ & $(0.38,0.30,0.19)$ & $(0.44,0.42,0.53)$ & $(0.46,0.39,0.53)$ & $(0.71,0.75,0.66)$ \\
$\ddot{A}_{2}$ & $(0.66,0.52,0.62)$ & $(0.48,0.51,0.49)$ & $(0.16,0.24,0.12)$ & $(0.17,0.33,0.35)$ \\
$\ddot{A}_{3}$ & $(0.61,0.73,0.74)$ & $(0.48,0.38,0.35)$ & $(0.42,0.51,0.40)$ & $(0.57,0.37,0.53)$ \\
$\ddot{A}_{4}$ & $(0.18,0.30,0.15)$ & $(0.57,0.64,0.58)$ & $(0.76,0.71,0.73)$ & $(0.35,0.42,0.39)$ \\
\hline
\end{tabular}

TABLE 5. Normalized 3-F decision matrix

Then we normalized the multi-polar decision matrix to get the normalized $m \mathrm{~F}$ decision matrix as shown in Table 5 by using (6.10).

Weighted Normalized $m \mathrm{~F}$ decision matrix is concluded (shown in Table 6) by using equation (6.11).

\begin{tabular}{ccccc}
\hline & $\ddot{C}_{1}$ & $\ddot{C}_{2}$ & $\ddot{C}_{3}$ & $\ddot{C}_{4}$ \\
\hline$\ddot{A}_{1}$ & $(0.12,0.07,0.05)$ & $(0.10,0.10,0.13)$ & $(0.09,0.11,0.12)$ & $(0.16,0.15,0.15)$ \\
$\ddot{A}_{2}$ & $(0.21,0.13,0.17)$ & $(0.11,0.13,0.12)$ & $(0.03,0.06,0.02)$ & $(0.03,0.06,0.08)$ \\
$\ddot{A}_{3}$ & $(0.19,0.18,0.20)$ & $(0.11,0.09,0.09)$ & $(0.08,0.14,0.09)$ & $(0.13,0.07,0.12)$ \\
$\ddot{A}_{4}$ & $(0.05,0.07,0.04)$ & $(0.13,0.16,0.15)$ & $(0.15,0.20,0.16)$ & $(0.08,0.08,0.08)$ \\
\hline
\end{tabular}

TABLE 6. Weighted normalized 3-F decision matrix

As $\ddot{C}_{1}$ and $\ddot{C}_{2}$ are cost criteria, and $\ddot{C}_{3}$ and $\ddot{C}_{4}$ are benefit criteria. Then the ( $m$ FPIS, $\left.A^{*}\right)$ and ( $m$ FNIS, $\left.A^{-}\right)$are given as follows,

$$
\begin{aligned}
& A^{*}=((0,0,0),(0,0,0),(1,1,1),(1,1,1)) \\
& A^{-}=((1,1,1),(1,1,1),(0,0,0),(0,0,0))
\end{aligned}
$$

Computing separation measure $\left(S_{j}^{*}\right.$ and $S_{j}^{-}$) between each attribute with ( $m$ FPIS, $A^{*}$ ) and ( $m$ FNIS, $A^{-}$) by using (6.12) and (6.13) respectively, we get the results (in Table 7),

\begin{tabular}{lcc}
\hline & $S^{*}$ & $S^{-}$ \\
\hline$\ddot{A}_{1}$ & 1.2387 & 1.2940 \\
$\ddot{A}_{2}$ & 1.3649 & 1.2123 \\
$\ddot{A}_{3}$ & 1.2841 & 1.2229 \\
$\ddot{A}_{4}$ & 1.2491 & 1.2885
\end{tabular}

TABLE 7. Separation Measures

Computing closeness coefficient C.Cof $f_{j}(j=1,2,3,4)$ of each alternative by using (6.14), we get results (shown in Table 8)

As $\ddot{A}_{1}=0.51$ is highest among others in Table 8 , which is the best ideal solution obtained via the multi-person TOPSIS process, so by the result obtained by the help of decisionmaking committee of the company is to invest money in a telecommunication company to get best results in earning money. 


\begin{tabular}{cc}
\hline & C.Cof \\
\hline$\ddot{A}_{1}$ & 0.51 \\
$\ddot{A}_{2}$ & 0.47 \\
$\ddot{A}_{3}$ & 0.48 \\
$\ddot{A}_{4}$ & 0.50
\end{tabular}

TABle 8. Closeness Coefficients

\section{Comparative Analysis}

$m \mathrm{IVF}$ set is a comprehensive idea that may be used to tackle real world problems are in the form of m-attributes expressed in interval valued rather than single-valued form. Existing theories cannot be utilized to address or investigate the issues; nevertheless, they do have limitations (see Table 9). Because of these restrictions, they are unable to deal with the data in form of multi-interval-valued data. In Table 9, our suggested model is compared to current methodologies. When the attributes have been studied as an interval-valued form with the decision taken by more than one person, these previous methods fail to execute. The proposed $m$ IVF addresses this shortcoming. It demonstrates that, in comparison to existing methods, our structure is sound and capable of successfully dealing with such challenges. Now, we talk about our proposed strategy and how precise it is.

\begin{tabular}{|c|c|c|}
\hline References & Disadvantage & Ranking \\
\hline Fuzzy Set [41] & fail to manage multipolar and interval-valued data & Unable to address \\
\hline Bipolar Fuzzy Set[26] & fail to manage multipolar and interval-valued data & Unable to address \\
\hline M-Polar Fuzzy Set [9] & fail to manage interval-valued data & Unable to address \\
\hline Interval-valued Fuzzy Set [20] & fail to manage multipolar data & Unable to address \\
\hline Proposed Method in this paper & Long calculations in decision-making & Addresses the multipolar and interval-valued data \\
\hline
\end{tabular}

TABLE 9. Superiority of $m$ IVF set over existing theories

\section{Conclusion And Recommendation}

Ordinarily, the problem of multipolar interval-valued fuzzy information occurs, and cannot be adequately elaborated using the current approaches. To overcome the uncertainty, a multipolar interval-valued fuzzy set is introduced, specifically in the interval-valued structure with multipolar. In this article, distance-based Sim.M with the multipolar intervalvalued set is used to improve the solution of many stressful situations. It broadens the range of applications in fields such as electronic optimization, industry, and forensic facial portraiture. All of Sim.M $>0.5$ and too much closeness of Sim.M ensures the importance of its application because it able more efficient and reliable the investigation agency to capture the person, one who is very near to the actual suspect (using $m$ IVF FORENSIC FACIAL PORTRAIT). In addition, based on similarity measure in MCDM, a new method for the best $m$ IVF alternatives is depicted. Furthermore, a TOPSIS technique is defined on the structure of $m \mathrm{IVF}$ for the selection of the best attribute. $m \mathrm{IVF}$ has defiantly opened 
the new ways to be applied in various hybrid structure of fuzzy sets such as $m$ IVF Soft set, $m I V$ Intuitionistic Fuzzy Soft Set, $m$ IV Neutrosophic Soft Set, $m$ IVF Hypersoft set, $m$ IVF Plithogenic Hypersoft Set, $m$ IV Intuitionistic Fuzzy Hypersoft Set, $m$ IV Neutrosophic Hypersoft Set, Pythagorean fuzzy uncertain environment, and their hybrid structures in the future. It can also be used in artificial intelligence, medical imaging, data mining, pattern recognition, social understanding, recommender frameworks, machine learning, social networks, signal processing, the monetary framework, neural networks, image processing, quantum geometry, and game theory, among other things.

\section{REFERENCES}

[1] A. Adeel, M. Akram, A. N. A. Koam, Group Decision-Making Based on m-Polar Fuzzy Linguistic TOPSIS Method, Symmetry, 11, No. 6 (2019) doi:10.3390/sym11060735

[2] M. Akram, m-Polar Fuzzy Labeling Graphs: Theory, Methods and Applications, In. (2019) 135-152, doi:10.1007/978-3-030-03751-2-4

[3] M. Akram, G. Ali, N. Alshehri, A New Multi-Attribute Decision-Making Method Based on m-Polar Fuzzy Soft Rough Sets, Symmetry, 9, No. 11 (2017) 271, doi:10.3390/sym9110271

[4] M. Akram, G. Ali, N. Waseem and B. Davvaz, Decision-making methods based on hybrid mF models, Journal of Intelligent and Fuzzy Systems35, No. 3 (2018) 3387-3403 doi:10.3233/JIFS-172282

[5] M. Akram, G. Shahzadi, (2016) Certain Characterization of m-Polar Fuzzy Graphs by Level Graphs, Punjab Uni. j. math. 49, No. 1 (2017) 10-12.

[6] M. Akram, N. Waseem. (2016) Certain Metrics in $m$-Polar Fuzzy Graphs, New Mathematics and Natural Computation, 12, No. 2 (2016)135-155 doi:10.1142/S1793005716500101

[7] M. Akram, N. Waseem and W. Dudek, Certain Types of Edge m-Polar Fuzzy Graphs, Iranian Journal of Fuzzy Systems,14, No. 4 (2017) doi:10.22111/ijfs.2017.3324

[8] M. Akram, N. Waseem and P. Liu P, Novel Approach in Decision Making with mPolar Fuzzy ELECTRE-I, International Journal of Fuzzy Systems, 21, No.4 (2019) 1117-1129, doi:10.1007/s40815-019-00608-y

[9] M. Akram and N. Waseem, Similarity Measure for new Hybrid Models: $m F$ sets and mF Soft Sets, Punjab Uni.j.maths. 51, No.6 (2019) 115-130

[10] K. T. Atanassov, Intuitionistic fuzzy sets, Fuzzy Sets and System, 20, No. 1(1986) 87-96, doi:10.1016/S01650114(86)80034-3

[11] I. Beg and T. Rashid, Intuitionistic Fuzzy Similarity Measure: Theory and Applications, Journal of Intelligent and Fuzzy Systems, 30, No. 2 (2016) 821-829, doi:10.3233/IFS-151805

[12] J. Chen, S. Li, S. Ma, X-P, Wang, m-Polar Fuzzy Sets: An Extension of Bipolar Fuzzy Sets, The Scientific World Journal (2014) :416530, doi:10.1155/2014/416530

[13] S. M. Chen, (1995) Measures of similarity between vague sets, Fuzzy Sets and Systems, 74, No. 1 (1995) 217-223, doi:10.1016/0165-0114(94)00339-9

[14] C. T. Chen, Extensions of the TOPSIS for group decision-making under fuzzy environment, Fuzzy Sets and Systems, 114, No. 1 (2000) 1-9, doi:10.1016/S0165-0114(97)00377-1

[15] De-Gang C, E.C. C. Tsang, D.S. Yeung, Some notes on the parameterization reduction of soft sets, In: Proceedings of the 2003 International Conference on Machine Learning and Cybernetics (IEEE Cat. No.03EX693), 1443 (2003) 1442-1445 doi:10.1109/ICMLC.2003.1259720

[16] M.B Gorzczany, A method of inference in approximate reasoning based on interval-valued fuzzy sets, Fuzzy Sets and Systems, 21, No. 1 (1987) 1-17, doi:10.1016/0165-0114(87)90148-5

[17] I. Grattan-Guinness, Fuzzy membership mapped onto interval and many-valued quantities, Mathematical Logic Quarterly, 22 (1976)149-160, doi:10.1002/malq.19760220120

[18] J.A. Goguen, L-fuzzy sets, Journal of Mathematical Analysis and Applications, 18(1967) 145-174 doi:10.1016/0022-247X(67)90189-8

[19] D. H. Hong and C. Kim, A note on similarity measures between vague sets and between elements, Information Sciences, 115, No. 1-4 (1999), doi:10.1016/S0020-0255(98)10083-X

[20] K. U. Jahn, Intervall-wertige Mengen, Mathematische Nachrichten, 68 (1975) 115-132, doi:10.1002/mana.19750680109 
[21] A. Kharal, Distance and Similarity Measures for Soft Sets, New Mathematics and Natural Computation, 6, No. 3 (2010) 321-334, doi:10.1142/S1793005710001724

[22] HAW-W, Khalifa, M. Saeed, M.K. Aslam, A. Mehmood, and S. S. Alodhaibi, Decision Making in Mass Media Using Fuzzy Soft Sets, Scientific Inquiry and Review, 4, No. 2 (2020) 63-77 doi:10.32350/sir.42.05

[23] S. C. Ming, M. S. Yeh, P. Y. Hsiao, A comparison of similarity measures of fuzzy values, Fuzzy Sets and Systems, 72, No. 1 (1995) 79-89 doi:10.1016/0165-0114(94)00284

[24] D.L. Olson, Comparison of weights in TOPSIS models, Mathematical and Computer Modelling, 40, No. 7-8 (2004) 721-727 doi:10.1016/j.mcm.2004.10.003

[25] I. Petrovic and M. Kankaras, A hybridized IT2FS-DEMATEL-AHP-TOPSIS multicriteria decision making approach: Case study of selection and evaluation of criteria for determination of air traffic control radar position, Decision Making: Applications in Management and Engineering, 3, No. 1 (2020) 146-164, doi:10.31181/dmame2003134p

[26] Wen-Ran, Zhang, Bipolar fuzzy sets and relations: a computational framework for cognitive modeling and multiagent decision analysis. In: NAFIPS/IFIS/NASA '94. Proceedings of the First International Joint Conference of The North American Fuzzy Information Processing Society Biannual Conference, The Industrial Fuzzy Control and Intellige (1994) 1305-309 doi:10.1109/IJCF.1994.375115

[27] K. Rashid and T. Rashid, Fuzzy logic model for the prediction of concrete compressive strength by incorporating green foundry sand, Computers and Concrete, 19, No. 6 (2017) 617-623, doi:10.12989/cac.2017.19.6.617 [28] M. Riaz and S. T. Tehrim, Certain Properties of Bipolar Fuzzy Soft Topology via Q-neighborhood, Punjab Uni.journal maths. 51, No.3 (2019) 113-131

[29] M. Riaz and M.R. Hashmi, Fuzzy Parameterized Fuzzy Soft Metric Spaces, Journal of Mathematical Analysis,9, No. (2018) 25-36

[30] M. Saeed, M. Zulqarnain and F. Dayan, Topsis Analysis for the Prediction of Diabetes Based on General Characteristics of Humans, International Journal of Pharmaceutical Sciences and Research, 9, No. 7 (2018) 2932-2939, doi:10.13040/IJPSR.0975-8232.9(7).2932-2939

[31] M. Saeed, Z. Anam, T. Kanwal, I. Saba, F. Memoona, M. Tabassum, Generalization of TOPSIS from Soft Set to Fuzzy Soft Sets in Decision Making Problem, Scientific Inquiry and Review, 1, No. 1 (2017) 11-18, doi:10.29145/sir/11/010102

[32] M. Saeed, Comparative Study of Airport Evaluation Problem by Using Fuzzy Soft Expert Set and AHP Technique, Science International (Lahore) 28 (2016) 2439-2443

[33] M. Saeed, M. Saqlain, A. Mehmood, Application of Similarity Measure on m-polar Interval-valued Neutrosophic Set in Decision Making in Sports, Neutrosophic Sets and Systems, 38 (2020) 317-334, doi:10.5281/zenodo.4300518

[34] M. Saeed, M. Saqlain, A. Mehmood, K. Naseer and S. Yaqoob, Multi-Polar Neutrosophic Soft Sets with Application in Medical Diagnosis and Decision-Making, Neutrosophic Sets and Systems, 33 (2020) 183-207, doi:10.5281/zenodo.3782895

[35] M. Saeed, A. Mehmood, A. Thabet and H. Saeed, Application of similarity measure in pattern recognition of covid-19 spread and its effects in Pakistan, Applied and Computational Mathematics, 20, No. 1 (2021) 108-123 [36] M. Saeed, A. Mehmood and A. Anwar, An extension of TOPSIS based on linguistic terms in triangular intuitionistic fuzzy structure, Punjab Uni. j. maths. 53, No. 6 (2021) 409-42, doi:1.52280/pujm.2021.530604

[37] M. Saqlain, N. Jafar and A. Riffat, Smart Phone Selection by Consumers in Pakistan: FMCGDM Fuzzy Multiple Criteria Group Decision Making Approach, Gomal University Journal of Research, 34, No. 1 (2018) 27-31

[38] H.S. Shih, H.J. Shyur, E.S. Lee, An extension of TOPSIS for group decision making, Mathematical and Computer Modelling, 45,No. 7-8 (2007) 801-813 doi:10.1016/j.mcm.2006.03.023

[39] D. Yong,Plant location selection based on fuzzy TOPSIS, An application of inteval valued fuzzy soft matrix (IVFSM) in decision making The International Journal of Advanced Manufacturing Technology, 28 (2006)839844, doi:10.1007/s00170-004-2436-5

[40] O. Yorulmaz, S.K. Yildirim, B.F. Yildirim, Robust Mahalanobis Distance based TOPSIS to Evaluate the Economic Development of Province, Operational Research in Engineering Sciences, Theory and Applications, 4 (2021) 102-123 doi:10.31181/oresta20402102y

[41] LA. Zadeh, Fuzzy Sets, Information and Control, 8, No. 3 (1965) 338-353 doi:10.1016/S00199958(65)90241-X 
[42] LA. Zadeh, The concept of a linguistic variable and its application to approximate reasoningI, Information Sciences, 8(1975) 199-249, doi:10.1016/0020-0255(75)90036-5

[43] S. H. Zolfani, M. Yazdani, D. Pamucar, and P. Zarate, A VIKOR and TOPSIS focused reanalysis of the MADM methods based on logarithmic normalization, Facta universitatis series: Mechanical Engineering,18(2020) 341355

[44] M. Zulqartnain and M. Saeed, An application of inteval valued fuzzy soft matrix (IVFSM) in decision making, Science International (Lahore), 28, No, 3 (2016) 2261-2264 